\title{
Editorial
}

\section{Computational Methods for Fracture 2015}

\author{
Timon Rabczuk, ${ }^{1,2}$ Xiaoying Zhuang, ${ }^{1,3}$ and Trung Nguyen-Thoi ${ }^{4}$ \\ ${ }^{1}$ Institute of Structural Mechanics, Bauhaus-Universität Weimar, Marienstraße 15, 99423 Weimar, Germany \\ ${ }^{2}$ School of Civil, Environmental and Architectural Engineering, Korea University, Republic of Korea \\ ${ }^{3}$ State Key Laboratory of Disaster Reduction in Civil Engineering, College of Civil Engineering, Tongji University, \\ Shanghai 200092, China \\ ${ }^{4}$ Division of Computational Mechanics, Ton Duc Thang University, Ho Chi Minh City, Vietnam \\ Correspondence should be addressed to Timon Rabczuk; timon.rabczuk@uni-weimar.de
}

Received 13 August 2015; Accepted 13 August 2015

Copyright (C) 2015 Timon Rabczuk et al. This is an open access article distributed under the Creative Commons Attribution License, which permits unrestricted use, distribution, and reproduction in any medium, provided the original work is properly cited.

Cracks are the progenitors of material failure. The success of devising better, lighter, and stronger materials relies also on how much we understand and how well we can predict and control fracture. Therefore, the modelling of fracture has always been a ubiquitous and foundational topic across engineering and sciences. From the early study of welding fracture of steel structures in the 1930s to nowadays the design and development of novel nanomaterials, researchers and engineers cannot emphasize more the power of simulation in assisting the improvement of materials performance. The rapid advances in computational methods for fracture, especially in the past two decades, have enhanced such power to the level where the prediction of complex fractures can be realized with multifields and models at different length scales being considered at the same time. This special issue is, as we hoped to share with readers, a showcase on the state-of-theart in the modelling of fractures.

The two key ingredients in the simulations of fractures are suitable physical models and computational methods. With the contributions from colleagues, we are glad to present this special issue addressing certain aspects of the abovementioned key ingredients: from the fundamental stress interaction analysis of multiple 3D cracks using complex variable functions to meshfree numerical methods for fracture propagation, from fractures in composites and piezoelectric materials to fractures in rocks and concrete, from static fracture modelling to dynamic fractures, and from pure mechanical problems to coupled field problems.
Multifield models for fracture propagation are an appealing topic in computational mechanics. In this special issue, models have been developed with special emphasis on rock mechanics and multifield problems in fracture with applications, for example, to coupled thermohydromechanical problems for compressed air energy storage and rock failure. Besides, the influence of fractures in functionally graded piezoelectric materials is investigated using meshfree methods. Special electromechanical-coupling enriched approximation near crack tip is devised. A review focusing on the state-of-the-art on developments in multiscale and multiphase modelling of hydraulic fracturing is carried out as well.

Composite structures are investigated including fractures in multilayered concrete/steel composite structures. The evaluation of fracture and delamination of the structure subjected to various jacking forces and ground conditions is studied and compared to the full-scale experimental results.

Dynamic fracture is in itself a wide field of study. Paper in this issue focuses on explicit dynamics and tackles, in particular, stochastic material parameter distribution and stabilized nodal integrated element-free Galerkin method for dynamic and implosive or explosive loading.

Fundamental solutions to curved and inclined 3D crack surfaces using complex stress functions are studied. The work features the curved length coordinate method and suitable quadrature rule is used to solve the integral equations which are compared with numerical examples. 
Through these studies, the issue presents a fair reflection of the current state-of-the-art, with a focus on modeling and simulation methods. It should inspire our colleagues with new ideas and trends for future research in the area.

Timon Rabczuk Xiaoying Zhuang Trung Nguyen-Thoi 


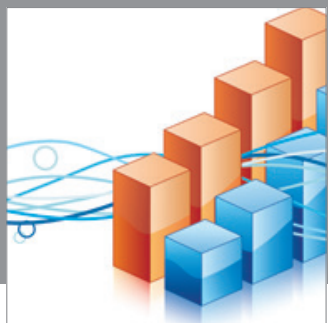

Advances in

Operations Research

mansans

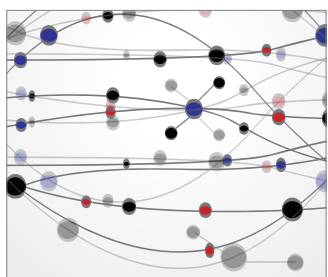

The Scientific World Journal
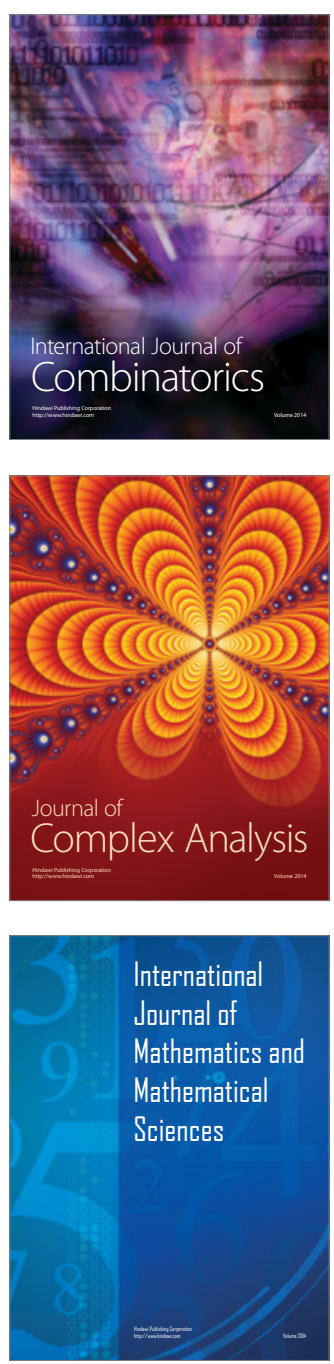
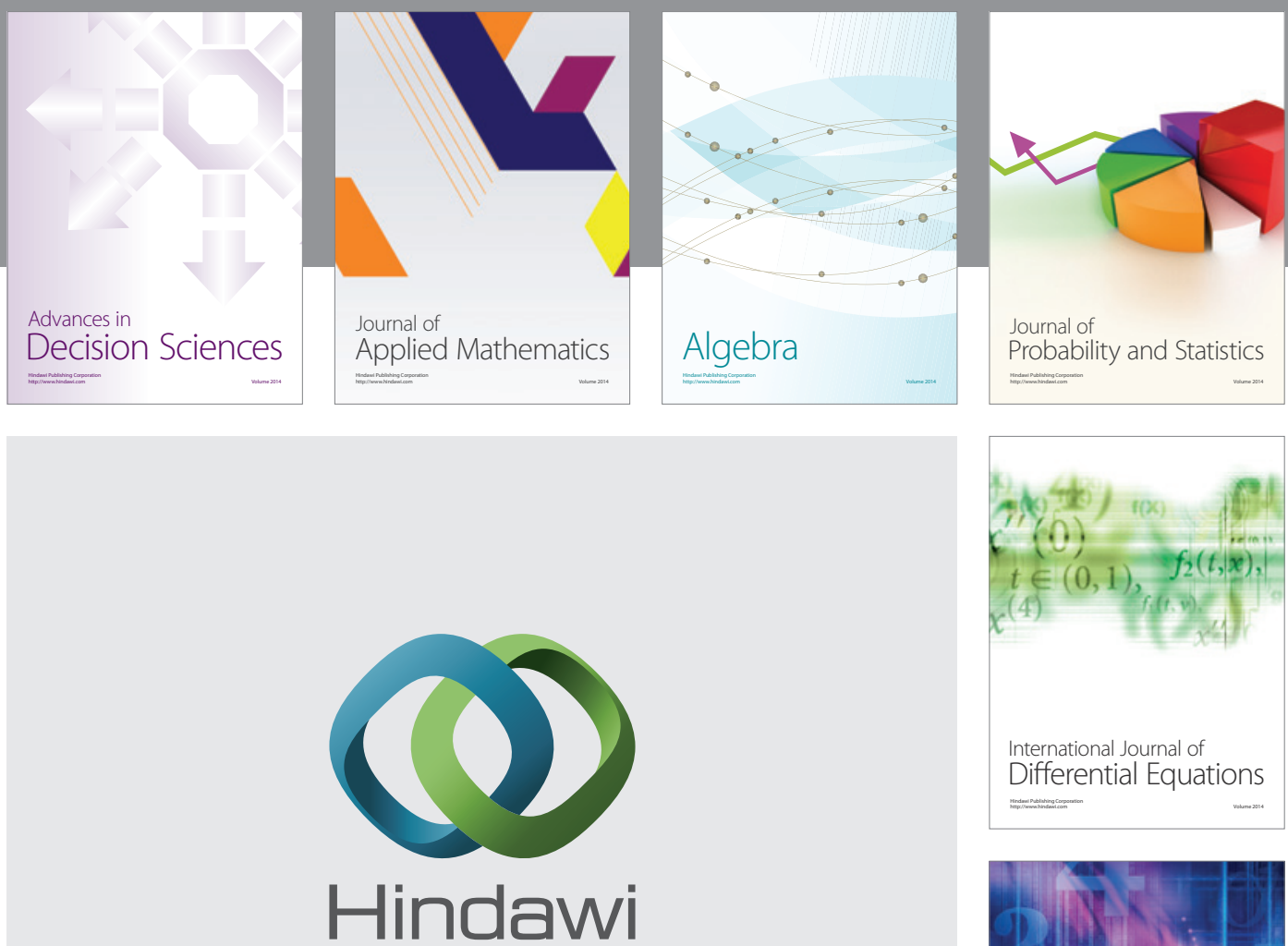

Submit your manuscripts at http://www.hindawi.com
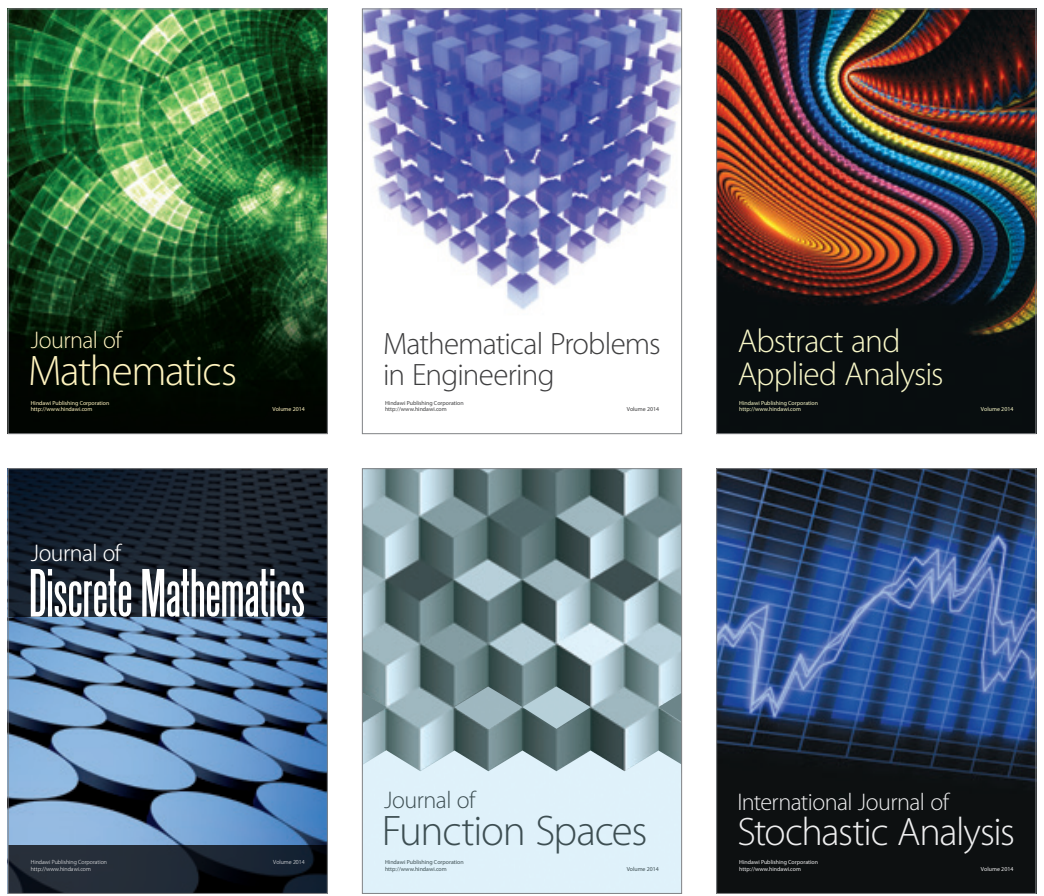

Journal of

Function Spaces

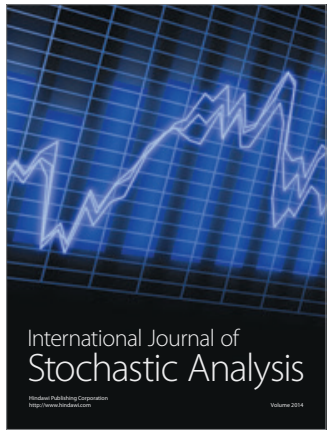

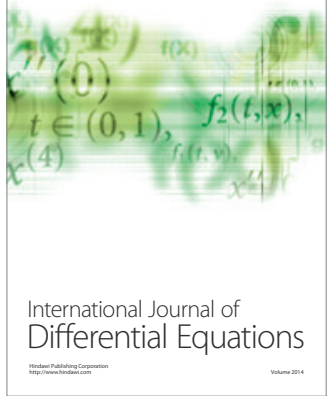
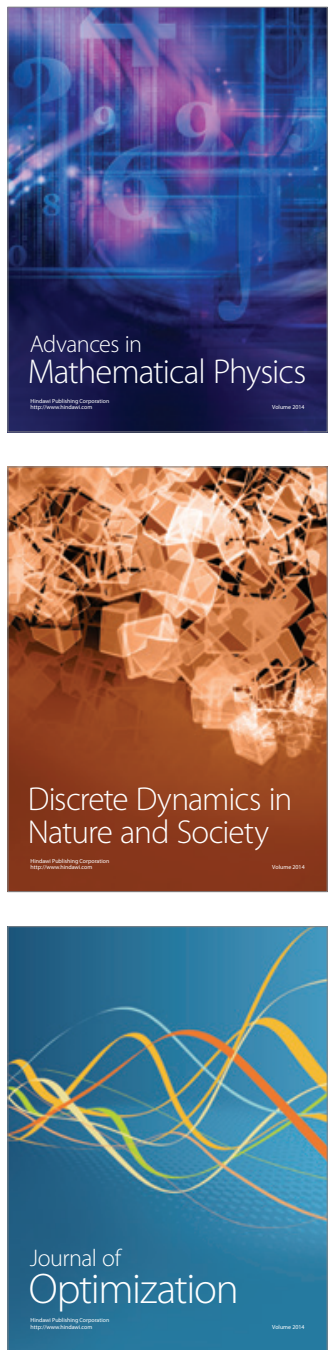